\title{
CONTRIBUCIÓN DEL LABORATORIO DE GEOMECÁNICA DE LA ECG-UCR AL ESTUDIO DE LOS DESLIZAMIENTOS EN COSTA RICA: LOS ÚLTIMOS 25 AÑOS
}

\author{
CONTRIBUTIONS FROM GEOMECHANICS LABORATORY (ECG-UCR) TO THE \\ STUDY OF LANDSLIDES IN COSTA RICA: THE PAST 25 YEARS
}

\author{
Rolando Mora \\ Escuela Centroamericana de Geología, Universidad de Costa Rica. \\ Apartado: 2142060 UCR, San Pedro, Montes de Oca, San José, Costa Rica \\ rmorach@gmail.con
}

(Recibido: 30/04/2014; aceptado: 24/09/2014)

\begin{abstract}
It is a brief journey through the last 25 years of his research on landslides, together with Geomechanics Laboratory of the Central American School of Geology. How incomprehensible resulted the Geomorphology for decision makers is emphasized, which led to develop advanced research on the instability of natural and manmade slopes. This research process has experienced continuous growth, which began with studies on susceptibility to landslides, grew up with the probabilistic analysis of landslide hazard and detailed studies, and does not stop, it has now initiated the investigation related to landslide risk. Despite having this kind of progress, our decision-makers are still making bad decisions, but this has not discouraged us, on the contrary, it has been allowed us to grow and so is intended to continue. Keywords: Landslides, geomechanics, geomorphology, natural disasters, Costa Rica.

RESUMEN: Se realiza un breve recorrido por los últimos 25 años de su investigación sobre los deslizamientos, del Laboratorio de Geomecánica de la Escuela Centroamericana de Geología. Se resalta lo incomprensible que resultó la Geomorfología para los tomadores de decisiones, lo cual motivó a desarrollar investigación avanzada en lo que respecta a la inestabilidad de las laderas y los taludes. Este proceso de investigación ha experimentado un crecimiento continuo, el cual se inició con los estudios de susceptibilidad a los deslizamientos, creció con el análisis probabilístico de la amenaza de deslizamiento y los estudios de detalle, y no se detiene, ahora se ha iniciado la investigación relacionada con el riesgo por deslizamiento. A pesar de contar con este tipo de avances, los tomadores de decisiones siguen tomando decisiones desacertadas, pero esta posición no ha causado desmotivación, por el contario ha permitido el crecimiento y así se pretende continuar.

Palabras clave: Deslizamientos, geomecánica, geomorfología, desastres naturales, Costa Rica
\end{abstract}




\section{INTRODUCCIÓN}

En este trabajo se pretende presentar una reseña del proceso seguido durante los últimos 25 años, en lo que respecta a la contribución del autor y de la Sección de Geotecnia e Hidrogeología, Escuela Centroamericana de Geología, Universidad de Costa Rica, al estudio de los procesos de deslizamiento en nuestro país.

En un inicio, se intentó desmostar la utilidad de las investigaciones geomorfológicas a nuestros tomadores de decisiones, sin embargo, este proceso resulto infructuoso, lo cual obligó a desarrollar y aplicar metodologías, de manera que la toma de decisiones basada en criterios eminentemente técnicos pueda ser una realidad.

Al final de este proceso hemos desarrollado toda una escuela de pensamiento alrededor del tema, con resultados muy importantes en el campo de la probabilidad de ocurrencia de deslizamientos, lo cual ha llevado a la conclusión de que se pueden hacer verdaderos estudios de amenaza de deslizamientos en nuestro país.

\section{GEOMORFOLOGÍA PRAGMÁTICA}

A finales de los 80 y principios de los 90 , se empezó con la utilización de la Geomorfología, aplicada de manera pragmática, es decir, analizando las formas del terreno desde una perspectiva genética y enfocada a la identificación de los procesos de la Geodinámica Externa que pueden representar una amenaza natural para la población, los desarrollos habitacionales y las obras de infraestructura. Lo anterior con la idea de tratar de que los tomadores de decisiones utilizaran, ante todo, criterios técnicos a la hora de decidir sobre permisos de construcción de proyectos de vivienda de interés social. Fue así como se desarrollaron proyectos de investigación en el sector comprendido entre el flanco sur del volcán Poás y la ciudad de Alajuela, en los valles de Ujarrás y Orosi, y los alrededores de Turrialba (Mora y Morales, 1988; Mora et al., 1989; Mora et al., 1990; Mora y Soto, 1990; Salazar et al. 1992). De particular relevancia fue el trabajo desarrollado en el valle de Orosi, en donde la subcuenca del río Jucó se constituyó en un laboratorio a escala natural (Fig. 1) (Mora, 2012), donde se dieron los primeros pasos en Costa Rica hacia una cuantificación de la amenaza por deslizamiento.

Al final de esta primera etapa, se comprendió que la Geomorfología no es muy entendible para los políticos tomadores de decisiones, o más bien, que estas personas no quieren o no les conviene comprenderla, sobre todo a la hora de decidir sobre otorgar o no permisos de construcción en áreas bajo amenaza. A raíz de este hecho, se toma la determinación de buscar otras formas de comunicar los resultados de nuestras investigaciones, pensando en tornarlas más cuantitativas, de manera que no se puedan presentar excusas por ignorancia geológica.

\section{SE INTENTA ALGO NUEVO: SUSCEPTIBILIDAD}

En 1994 se publica el primer mapa de susceptibilidad a los deslizamientos del Gran Área Metropolitana de Costa Rica (Mora et al., 1994), el cual utiliza la metodología conocida como Mora-Vahrson. Este tipo de abordaje se extendió a lugares como la Península de Papagayo (Mora et al., 2002), el Cantón de San José (Mora, 2004), el Bajo Cacao (Atenas) (Mora, 2009) y algunos otros lugares de nuestro país. La metodología original fue modificada para poder ser aplicada en lugares donde la escases de información geológica representa un gran obstáculo, incluyéndose una variable geomorfológica en sustitución de la información geológica requerida por la metodología original (Camacho et al., 2004).

Curiosamente la metodología ha encontrado una aceptación muy buena fuera de Costa Rica, como lo muestran los ejemplos de su aplicación en El Salvador (SNET, 2014), Nicaragua (INETER, 2014), Guatemala (GEOPETROL S.A., 2007) e Irán (Koorkinejad \& Gorgani, 2007), sin embargo, en nuestro país continúa sin adoptarse como una metodología oficial, en su lugar se adoptan oficialmente índices que no han sido exhaustivamente evaluados, ni publicados en revistas científicas o la INTERNET. Tomando en consideración 


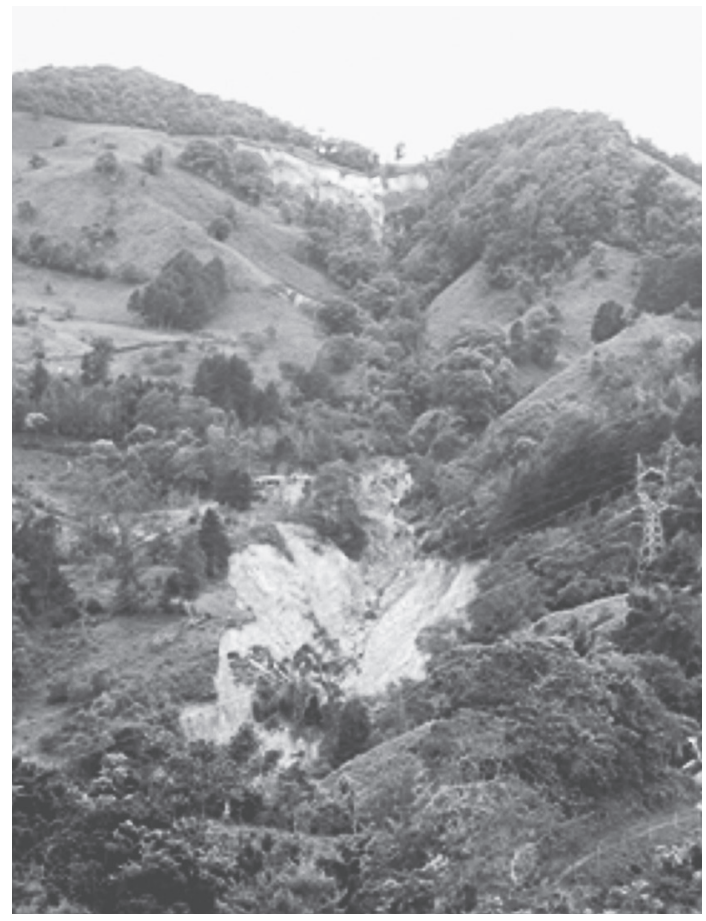

Fig. 1: Deslizamientos en la parte alta de la sub-cuenca del río Jucó, Orosi, Costa Rica.

el éxito alcanzado en el exterior y la poca relevancia dada al método en nuestro país, podemos dar como valedero el proverbio que dice: "nadie es profeta en su propia tierra".

\section{AMENAZA EN TÉRMINOS PROBABILÍSTICOS}

Definitivamente había que seguir investigando y tratando de que la temática se tomara en cuenta de forma apropiada por los tomadores de decisiones. Fue así como la mirada se tornó hacia la evaluación de la amenaza en términos probabilísticos. Primero, se inscribió un proyecto de investigación ante la Vicerrectoría de Investigación de la Universidad de Costa Rica con el código 113-A5-110, con una duración de tres años, el cual se desarrolló en la subcuenca del río Jucó, en el Valle de Orosi, Cantón de Paraíso, Cartago. Este lugar se llegó a convertir en un laboratorio a escala natural para el modelamiento de los procesos de deslizamiento.
Durante la ejecución del proyecto, se exploraron opciones para modelar la amenaza por deslizamiento, llegándose a la conclusión de que la forma más apropiada de hacerlo consistía en la aplicación de metodologías basadas en el muestreo aleatorio de distribuciones probabilísticas de los parámetros que forman parte de la ecuación del talud infinito. Fue así como se adquirió la licencia del programa PISA (Haneberg, 2005), el cual ejecuta cálculos probabilísticos de estabilidad de taludes, en condiciones estáticas y pseudoestáticas, a partir de modelos de elevación digital del terreno, utilizando la ecuación del talud infinito $\mathrm{y}$ funciones de probabilidades para cada uno de los parámetros que la integran.

La metodología se utilizó por primera vez en nuestro país en la sub-cuenca del río Jucó (Mora, 2007b), posteriormente se aplicó en otro sector del Valle de Orosi (Mora, 2007a). Con estas aplicaciones se logró implementar el protocolo de aplicación de la metodología como quehacer rutinario en el Laboratorio de Geomecánica de la Escuela Centroamericana de Geología, lo cual permitió desarrollar labores de acción social, investigación y captación de fondos externos mediante el análisis de casos en el Bajo Anonos de Escazú Mora, 2010a), la cuenca del río Cruz en Escazú (Mora \& Granados, 2014) (Fig. 2) y la comunidad de Mansiones de Montes de Oca (Granados , 2013).

\section{ESTUDIO A DETALLE CON MÉTODOS DETERMINÍSTICOS}

Además del enfoque regional que se le ha dado a la susceptibilidad y a la probabilidad de ocurrencia de deslizamientos, se han desarrollado estudios determinísticos de estabilidad de laderas en casos donde se ha requerido. Estos estudios involucran la confección de perfiles topográficos, exploración geotécnica de campo, ensayos de laboratorio, modelamiento geotécnico con programas de cómputo y elaboración de propuestas para la remediación.

Algunos de los casos estudiados que deben mencionarse son Mansiones de Montes de Oca 


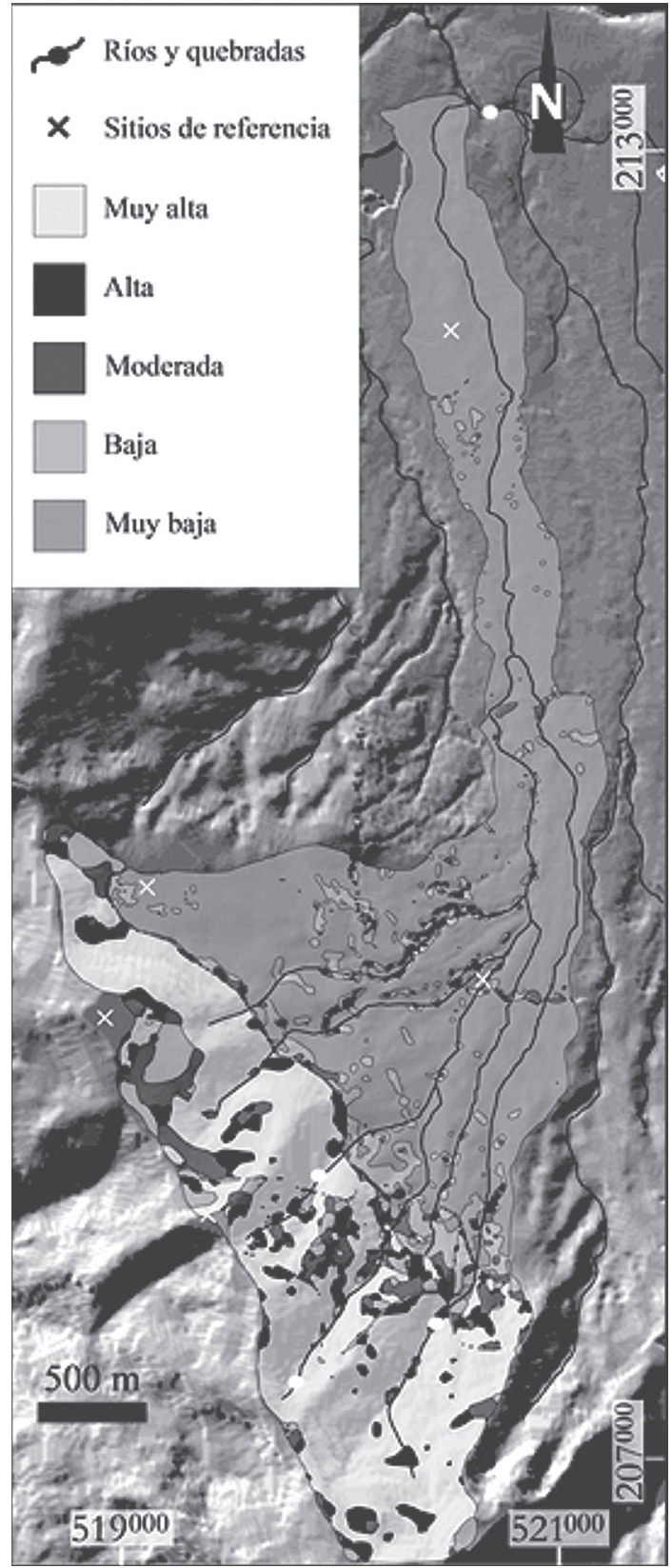

Fig. 2: Probabilidad de ocurrencia de deslizamientos en la cuenca del río Cruz, Escazú (Mora \& Granados , 2014).
(Mora \& Granados, 2012), la cárcel del Buen Pastor en San Rafael Arriba de Desamparados (Mora, 2010b), Urbanización El Río en Heredia (Mora, 2008), entre muchos otros analizados para diversas personas físicas y jurídicas.

En los últimos años se ha incursionado en el estudio de la amenaza por caída de rocas, mediante la simulación de trayectorias de bloques de roca inestables en San Gabriel de Aserrí y en Bebedero de Escazú (González \& Mora, 2013).

\section{COMENTARIO FINAL}

Lo incomprensible que resulta la Geomorfología para nuestros tomadores de decisiones nos motivó a desarrollar investigación avanzada en lo que respecta a la inestabilidad de las laderas y los taludes.

Los estudios se inciaron con un componente básico de susceptibilidad a los deslizamientos y posteriormente se pasó a análisis probabilísticos de la amenaza, lo que posteriormente se traducirá en una investigación relacionada con el riesgo por deslizamiento.

\section{REFERENCIAS BIBLIOGRÁFICAS}

CAMACHO, D., CHAVES, J. \& MURILLO, D., 2004: Diagnóstico de la susceptibilidad al deslizamiento e inundación, análisis del contexto sísmico y reconocimiento de peligros volcánicos para el Cantón de Montes de Oca: una contribución al plan regulador del Municipio.- 106 págs. Univ. de Costa Rica, San José [Seminario de Graduación].

GEOPETROL S.A., 2007: Estudio hidro-geológico para la implementación de un sistema de monitoreo y alerta ante deslizamientos 
en asentamientos urbanos del departamento de Guatemala, Centro América.- http:// desastres.usac.edu.gt/documentos/docgt/ pdf/spa/doc0134/doc0134-parte01.pdf [Consulta: 18 de marzo de 2014].

GONZÁLEZ, A \& MORA, R 2013: Evaluación del Peligro de Caída de Rocas Alrededor del Liceo de San Gabriel de Aserrí San José.- 25 págs. Escuela Centroamericana de Geología, Univ. de Costa Rica [Inf. interno].

GRANADOS , R., 2013: Determinación de la probabilidad de ocurrencia de deslizamiento en suelos derivados de cenizas volcánicas, Mansiones, San Rafael, Montes de Oca, Costa Rica.- 202 págs. Universidad de Costa Rica, San José [Tesis Lic.].

HANEBERG, W., 2005: PISA: Probabilistic Infinite Slope Analysis. User Manual.- 15 págs. Haneberg Geo-science, California, USA.

INETER, 2014: República de Nicaragua, Mapa de Susceptibilidad a Deslizamientos.- http:// webserver2.ineter.gob.ni/geofisica/desliza/ desli.html [Consulta: 18 de marzo de 2014].

KOORKINEJAD, M. \& GORGANI, M. T., 2007: Applicability of Mora-Vahrson landslide hazard zonation model in Ciarrodbar watershed, Iran.- Proceedings of the EGU General Assembly, Viena (15-20 April): 602-603.

MORA, R., 2004: Proyecto Habitacional Orokay, Orosi, Paraíso, Cartago: construyendo la vulnerabilidad física de las viviendas y sus moradores.- 5 págs., Escuela Centroamericana de Geología, Universidad de Costa Rica [Inf. interno].
MORA, R., 2004: Evaluación de la susceptibilidad al deslizamiento del Cantón de San José, Provincia de San José, Costa Rica.14 págs. Escuela Centroamericana de Geología, Univ. de Costa Rica, San José [Inf. interno].

MORA, R., 2007a: Geomorfología y probabilidad de ocurrencia de deslizamientos, Proyecto Eco-Vivienda, Orosi, Paraíso, Cartago.Memorias del IX Seminario Nacional de Geotecnia, San José, Costa Rica: 201-213

MORA, R., 2007b: Probabilistic analysis of slope stability, Jucó river sub-basin, Paraíso, Cartago, Costa Rica.- Proceedings of the $32^{\text {nd }}$ International Symposium on Remote Sensing of the Environment, San José, Costa Rica [Disco compacto sin paginación]

MORA, R., 2008: Estudio de estabilidad del talud oeste, Urbanización El Río, Heredia.10 págs., Fundación de la Universidad de Costa Rica para la Investigación (FUNDEVI) [Inf. interno].

MORA, R., 2009: Aplicación de la metodología MVM para determinar la susceptibilidad a deslizamientos en el Bajo Cacao, Atenas, Alajuela.- 17 págs. Escuela Centroamericana de Geología, Univ. de Costa Rica [Inf. interno].

MORA, R., 2010a: Estabilidad en condiciones estáticas y probabilidad de ocurrencia de deslizamientos en condiciones pseudo-estáticas de las laderas del Bajo Los Anonos, San Rafael, Escazú, San José.- Ingeniería, 20(1/2): 41-49.

MORA, R., 2010b: Propuesta de estabilización del talud localizado al costado oeste 
de la cárcel del Buen Pastor, San Rafael Arriba, Desamparados.- 8 págs. Escuela Centroamericana de Geología, Univ. de Costa Rica [Inf. interno].

MORA, R., 2012: Geomorfología de la subcuenca del río Jucó, Orosi, Paraíso, Cartago, Costa Rica.- En: ELLIS, J., GUARDADO, R. \& SÁNCHEZ, I., (eds): Deslizamientos: Efectos, evaluación y gestión del riesgo.- Ed. UNESCO, Quito: 214-225.

MORA, R. \& GRANADOS, R., 2012: Análisis de estabilidad de taludes considerando la incertidumbre de los datos: caso comunidad de Mansiones de Montes de Oca, San José, Costa Rica.- Rev. Geol. Amér. Central, 47: 133-141.

MORAR. \& GRANADOS, R., 2014: Zonificación de los procesos denudacionales en la micro-cuenca del río Cruz, Escazú, San José, Costa Rica: recomendaciones para mejorar la gestión del territorio.- Rev. Geol. Amér. Central, 50: 99-112.

SALAZAR, L.G., OBANDO, L. \& MORA, R., 1992: Acueducto Metropolitano, Tramo embalse El Llano-Río Navarro (Costa Rica): Un sitio bajo amenaza.- Rev. Geol. Amér. Central, 14: 85-96.

\section{SERVICIO NACIONAL DE ESTUDIOS} TERRITORIALES (SNET), 2014: Mapa de susceptibilidad por remoción en masa de suelos y rocas.- http://www.snet.gob. sv/ver/geologia/amenaza+deslizamientos/ [Consulta: 18 de marzo de 2014].
MORA, R. \& MORALES, L.D., 1988: La Susceptibilidad a la Inestabilidad de Laderas en la Zona Norte de Alajuela y el Terremoto de Fraijanes de 1888. Efectos y Previsión.- Rev. Geogr. Amér. Central, 25/26: 311-322.

MORA, R., SARAVIA, M., CHAVARRÍA, R. \& LEANDRO, F., 1989: Análisis Preliminar de Estabilidad de Laderas, Valle de Ujarrás, Paraíso Cartago, Costa Rica.-GeoIstmo, 3(2): 61-69.

MORA, R., CERDAS, A., MOLINA, F. \& VEGA, E., 1990: Caracterización Geológica del Deslizamiento Chiz (Turrialba, Provincia de Cartago, Costa Rica): Amenaza y Prevención. Rev. Geol. Amér. Central, 11: 59-68.

MORA, R. \& SOTO, G., 1990: Landslide and avalanche on Itiquís, Alajuela, Costa Rica.- Landslides News, 4: 170-172.

MORA, R., MORA, S. \& VAHRSON, W.G., 1994: Mapa de Amenaza de Deslizamientos, Valle Central, Costa Rica.- Escala 1:250 000.- Centro de Coordinación para la Prevención de Desastres Naturales de América Central (CEPREDENAC), San José.

MORA, R., CHAVES, J. \& VÁZQUEZ, M., 2002: Zonificación de la Susceptibilidad al Deslizamiento: resultados obtenidos para la Península de Papagayo mediante la modificación del método Mora-Vahrson.- III Curso Internacional sobre Microzonificación y su Aplicación en la Mitigación de Desastres. Lima, Perú: 38-46. 